\title{
Semantic methodology in Huge-Scale Storage Systems
}

\author{
Bindusri G.1)
}

\begin{abstract}
The difficulties of taking care of the dangerous development in information volume and unpredictability cause the expanding requirements for semantic inquiries. The semantic inquiries can be deciphered as the relationship mindful recovery, while containing surmised comes about. Existing distributed storage systems for the most part neglect to offer a satisfactory capacity for the semantic inquiries. Since the genuine esteem or worth of information intensely relies on upon how productively semantic hunt can be completed on the information in (close) ongoing, expansive portions of information wind up with their qualities being lost or fundamentally lessened because of the information staleness. To address this issue, we propose a close ongoing and financially savvy semantic questions based strategy, called FAST. The thought behind FAST is to investigate and misuse the semantic connection inside and among datasets by means of relationship mindful hashing and sensible level organized tending to altogether lessen the handling inactivity, while acquiring acceptably little loss of information pursuit precision. The close continuous property of FASTenables quick recognizable proof of connected documents and the critical narrowing of the extent of information to be handled. FASTsupports a few sorts of information examination, which can be executed in existing searchable stockpiling systems. We direct a certifiable utilize case in which youngsters revealed missing in a to a great degree swarmed environment (e.g., an exceedingly prevalent grand spot on a pinnacle vacationer day) are recognized in an opportune manner by dissecting 60 million pictures utilizing FAST. Quick is further enhanced by utilizing semantic-mindful namespace to give alterable and versatile namespace administration for ultra-substantial capacity frameworks. Broad test comes about show the effectiveness and viability of FAST in the execution upgrades.
\end{abstract}

Keywords : cloud storage, data analytics, real-time performance, semantic correlation, storage, FAST.

\section{Introduction}

Cloud storage systems for the most part contain a lot of information that basically require quick and exact information recovery to support intllegent and adaptive cloud administrations [1-3]. For instance, 7 percent of shoppers put away their substance in the cloud in 2011, and the figure will develop to 36 percent in 2016, as per the Gartner, Inc.[4-6] and Storage Newsletter[7-9] reports. Normal stockpiling limit per family unit will develop from 464 Gigabytes in 2011 to 3.3 Terabytes in 2016. Up until this point, just a minor division of the

\footnotetext{
Received(September 11, 2015), Review Result(1st: September 25, 2015, 2nd: October 22, 2015), Accepted(December 10, 2015)

${ }^{1}$ (Corresponding Author) Vidyaa Vikas Educational Institutions, Mysore - Bannur Road, Alanahally, Mysuru, Karnataka, India email: Bindusri8089@gmail.com
} 
information being created has been investigated for their potential values using information examination (DA) apparatuses. IDC gauges that by 2020, as much as 33 percent of all information will contain data that may be important if examined[10-13]. Henceforth, effective information investigation are essential.

Existing content-based examination apparatuses cause high complexity and expenses, as well as neglect to adequately deal with the monstrous measures of documents. The high multifaceted nature routinely prompts to moderate preparing operations and high and regularly unsuitable inertness. Because of the unsatisfactory idleness, the staleness of information extremely decreases the estimation of information. The value or estimation of information with regards to information investigation implies the important learning covered up in the information that can specifically convert into monetary qualities/picks up in business knowledge applications or new logical revelations in logical applications. Since the esteem/worth of information commonly lessens with time, a lot of information are frequently rendered futile, albeit exorbitant assets, for example, calculation, stockpiling and system transfer speed, have as of now been devoured to create, gather or potentially prepare these information. Hence, we argue that (near-) real-time schemes are basic to getting profitable information in searchable data analytics[14-16].

\section{System Analysis}

\subsection{Related Work}

In this area, we display a brief study of late reviews in the writing most pertinent to the FAST research from the parts of information investigation, searchable record systems and deduplication-based redundancy detection.

Data analytics. Data analytics has gotten expanding consideration from both industrial and academic communities. So as to scaffold the semantic hole between the low-level information substance and the abnormal state client comprehension of the system, a conduct based semantic investigation system is proposed, which incorporates an examination motor for extricating examples of client determined conduct models. ISABELAQA is a parallel inquiry preparing motor that is planned and upgraded for dissecting and handling spatiotemporal, multivariate logical information. MixApart utilizes a coordinated information reserving and booking answer for permit MapReduce calculations to break down information put away on big business stockpiling systems. The frontend reserving layer empowers the nearby stockpiling execution 
required by information examination. The common stockpiling back-end improves information administration. Three normal examination systems, including topological investigation, illustrative measurements, and perception, are investigated to bolster productive information development between in-situ and in-travel calculations. In this specific circumstance, FAST is a valuable instrument that supplements and enhances the current plans to get connected partiality from close copy pictures and execute semantic gathering to support quick query service.

Searchable file systems. Spyglass[17-22] exploits the region of record namespace and skewed appropriation of metadata to outline namespace progression into a multi-dimensional K-D tree and uses multilevel forming and parceling to look after consistency. SmartStore[23-26] utilizes idle semantic ordering (LSI) apparatus to total semantically corresponded documents into gatherings and bolster complex inquiries. Dissimilar to these methodologies, FAST offers the notable components of questioning close copy pictures in a real-time manner.

\subsection{Existing System}

ISABELAQA is a parallel inquiry handling motor that is designed and optimized for examining and preparing spatiotemporal, multivariate logical information. MixApart utilizes an incorporated information reserving and planning answer for permit MapReduce computations to dissect information put away on enterprise storage systems.

The frontend caching layer empowers the nearby stockpiling execution required by information examination. The common storage back-end streamlines information administration.

Spyglass misuses the region of document namespace and skewed conveyance of metadata to delineate namespace chain of command into a multi-dimensional K-D tree and uses multilevel forming and dividing to look after consistency.

Glance, a just-in-time sampling-based system, can give precise responses to total and top-k questions without earlier information.

\subsection{Disadvantages of Existing System}

Existing content-based examination devices cause high many-sided quality and expenses, as well as neglect to successfully deal with the monstrous measures of records.

The high complexity routinely prompts to moderate handling operations and high and frequently inadmissible idleness. Because of the unsatisfactory inactivity, the staleness of information seriously lessens the estimation of information. 
Existing ways to deal with unstructured information look and investigation depend on either framework based lumps of information records.

Due to the long latency acquired in information preparing and the subsequent information staleness, the esteem/worth of information gets to be distinctly lessened and in the long run invalidated.

\subsection{Proposed System}

In the setting of this paper, searchable information investigation are interpreted as getting information value/worth through questioned results, for example, finding a profitable record, a corresponded procedure ID, a critical picture, a reconstruct system log, and so on.

We propose a novel near-real-time methodology for analyzing massive data, called FAST, with an outline objective of proficiently preparing such information in a constant way.

The key thought behind FAST is to investigate and abuse the connection property inside and among datasets by means of enhanced relationship mindful hashing and level organized tending to fundamentally lessen the preparing inactivity of parallel questions, while acquiring acceptably little loss of exactness.

The approximate plan for constant execution has been broadly perceived in framework outline and top of the line registering. Generally, FAST goes past the basic blend of existing procedures to offer productive information examination through altogether expanded handling speed. Through the investigation of the FAST system, we intend to make the accompanying commitments for close real-time data analytics.

\section{Conclusion}

This paper proposes a close real-time scheme, called FAST, to support proficient and cost-effective searchable data analytics in the cloud. Quick is intended to abuse the relationship property of information by utilizing connection mindful hashing and sensible level organized tending to. This empowers FAST to altogether lessen handling idleness of associated record identification with acceptably little loss of precision. We talk about how the FAST technique can be identified with and used to improve some stockpiling frameworks, including Spyglass and SmartStore, and also an utilization case. Quick is exhibited to be a helpful instrument in supporting near real-time processing of real-world data analytics applications. 


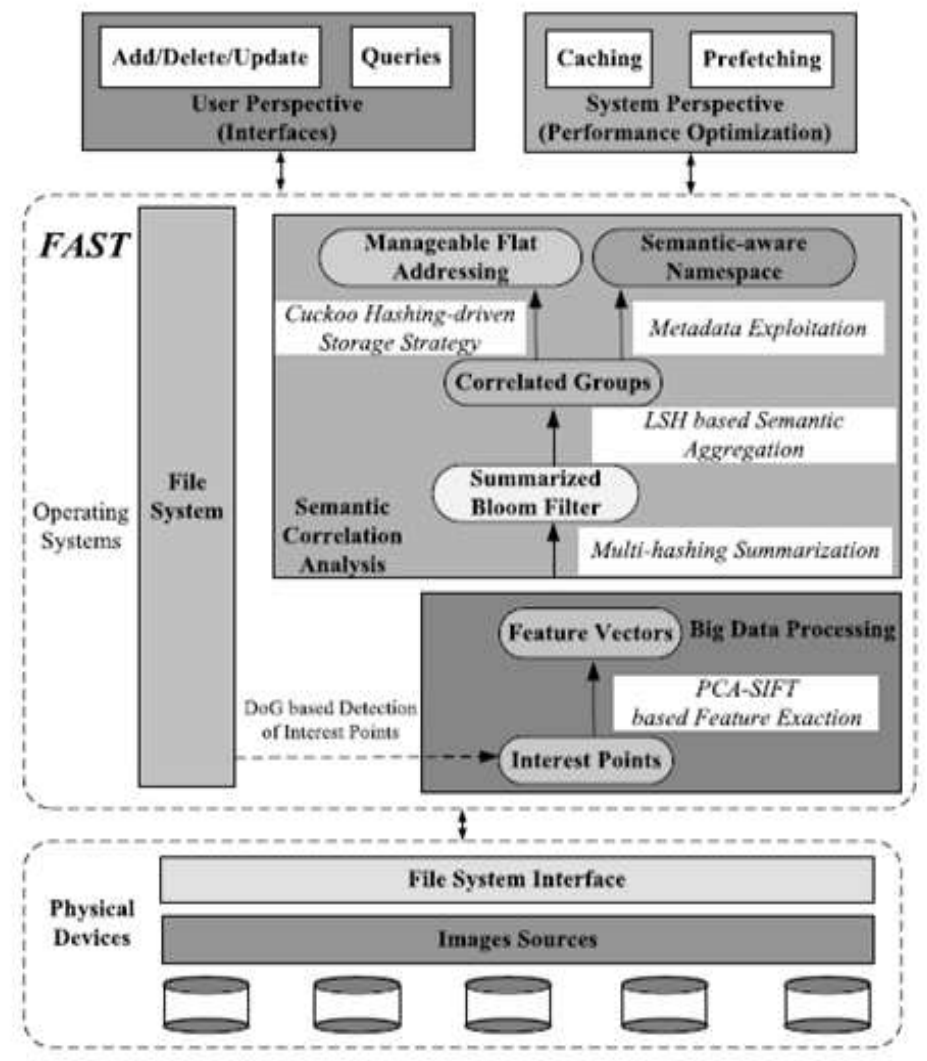

[Fig. 1] System Architecture

\section{References}

[1] M. Armbrust, A. Fox, R. Griffith, A. D. Joseph, R. Katz, A. Konwinski, G. Lee, D. Patterson, A. Rabkin, I. Stoica, and M. Zaharia, A view of cloud computing, Commun. ACM, (2010), Vol.53, No.4, pp.50-58.

[2] A. Marathe, R. Harris, D. K. Lowenthal, B. R. de Supinski, B. Rountree, M. Schulz, and X. Yuan, A comparative study of highperformance computing on the cloud, in Proc. 22nd Int. Symp. High-Perform. Parallel Distrib. Comput., (2013), pp.239-250.

[3] P. Nath, B. Urgaonkar, and A. Sivasubramaniam, Evaluating the usefulness of content addressable storage for high-performance data intensive applications, in Proc. 17th Int. Symp. High-Perform. Parallel Distrib. Comput., (2008), pp.35-44.

[4] Gartner, Inc., Forecast: Consumer digital storage needs, 2010 - 2016, (2012).

[5] Storage Newsletter, 7\% of consumer content in cloud storage in 2011, 36\% in 2016, (2012). 
[6] J. Gantz and D. Reinsel, The digital universe in 2020: Big data, bigger digital shadows, and biggest growth in the far east, International Data Corporation (IDC) iView, (2012) December.

[7] Y. Hua, W. He, X. Liu, and D. Feng, SmartEye: Real-time and efficient cloud image sharing for disaster environments, in Proc. INFOCOM, (2015), pp.1616-1624.

[8] Y. Ke and R. Sukthankar, PCA-SIFT: A more distinctive representation for local image descriptors, in Proc. IEEE Conf. Comput. Vis. Pattern Recog., (2004), pp.506-513.

[9] Y. Ke, R. Sukthankar, and L. Huston, Efficient near-duplicate detection and sub-image retrieval, in Proc. ACM Multimedia, (2004), pp.869-876.

[10] J. Liu, Z. Huang, H. T. Shen, H. Cheng, and Y. Chen, Presenting diverse location views with real-time near-duplicate photo elimination, in Proc. 29th Int. Conf. Data Eng., (2013), pp.505-506.

[11] D. Zhan, H. Jiang, and S. C. Seth, CLU: Co-optimizing locality and utility in thread-aware capacity management for shared last level caches, IEEE Trans. Comput., (2014), Vol.63, No.7, pp.1656-1667.

[12] P. Indyk and R. Motwani, Approximate nearest neighbors: towards removing the curse of dimensionality, in Proc. 13thAnnu.ACMSymp.TheoryComput., (1998), pp.604-613.

[13] R. Pagh and F. Rodler, Cuckoo hashing, in Proc. Eur. Symp. Algorithms, (2001), pp.121-133.

[14] Y. Hua, H. Jiang, Y. Zhu, D. Feng, and L. Xu, "SANE: Semanticaware namespace in ultra-large-scale file systems," IEEE Trans. Parallel Distrib. Syst., (2014), Vol.25, No.5, pp.1328-1338.

[15] http://www.changewaveresearch.com/, Changewave research, (2011).

[16] Science Staff, Dealing with data-Challenges and opportunities, Science, (2011), Vol.331, No.6018, pp.692-693.

[17] X. Tan, S. Chen, Z.-H. Zhou, and F. Zhang, Face recognition from a single image per person: A survey, Pattern Recog., (2006), Vol.39, No.9, pp.1725-1745.

[18] T. Ahonen, A. Hadid, and M. Pietikainen, Face description with local binary patterns: Application to face recognition, IEEE Trans. Pattern Anal. Mach. Intell., (2006), Vol.28, No.12, pp.2037-2041.

[19] X. Tan and B. Triggs, Enhanced local texture feature sets for face recognition under difficult lighting conditions, IEEE Trans. Image Process., (2010), Vol. 19, No. 6, pp.1635-1650.

[20] J. Wright, A. Y. Yang, A. Ganesh, S. S. Sastry, and Y. Ma, Robust face recognition via sparse representation, IEEE Trans. Pattern Anal. Mach. Intell., (2009), Vol.31, No.2, pp.210-227.

[21] Y. Hua and X. Liu, Scheduling heterogeneous flows with delayaware deduplication for avionics applications, IEEE Trans. Parallel. Distrib. Syst., (2012), Vol.23, No.9, pp.1790-1802.

[22] A. W. Leung, M. Shao, T. Bisson, S. Pasupathy, and E. L. Miller, Spyglass: Fast, scalable metadata search for large-scale storage systems, in Proc. 7th USENIX Conf. File Storage Technol., (2009), pp.153-166.

[23] Y. Hua, H. Jiang, Y. Zhu, D. Feng, and L. Tian, SmartStore: A new metadata organization paradigm with semantic-awareness for next-generation file systems, in Proc. Int. Conf. High Perform. Comput. Netw. Storage Anal., (2009), pp.1-12. 
[24] E. Riedel, M. Kallahalla, and R. Swaminathan, A framework for evaluating storage system security, in Proc. USENIX Conf. File Storage Technol., (2002), pp.15-30.

[25] S. Kavalanekar, B. Worthington, Q. Zhang, and V. Sharda, Characterization of storage workload traces from production Windows servers, in Proc. IEEE Int. Symp. Workload Characterization, (2008), pp.119-128.

[26] D. Ellard, J. Ledlie, P. Malkani, and M. Seltzer, Passive NFS tracing of email and research workloads, in Proc. USENIX Conf. File Storage Technol., (2003), pp.203-16. 